\title{
Reversal of axonal loss and disability in a mouse model of progressive multiple sclerosis
}

\author{
Alexandre S. Basso, ${ }^{1}$ Dan Frenkel, ${ }^{1,2}$ Francisco J. Quintana, ${ }^{1}$ Frederico A. Costa-Pinto, ${ }^{3}$ \\ Sanja Petrovic-Stojkovic, ${ }^{1}$ Lindsay Puckett, ${ }^{1}$ Alon Monsonego, ${ }^{4}$ Amnon Bar-Shir, ${ }^{5}$ Yoni Engel, ${ }^{5}$ \\ Michael Gozin, ${ }^{5}$ and Howard L. Weiner ${ }^{1}$
}

${ }^{1}$ Center for Neurologic Diseases, Brigham and Women's Hospital, Harvard Medical School, Boston, Massachusetts, USA. 2Department of Neurobiochemistry, George S. Wise Faculty of Life Sciences, Tel Aviv University, Tel Aviv, Israel. ${ }^{3}$ Department of Pathology, Faculty of Veterinary Medicine and Animal Science, University of São Paulo, São Paulo, Brazil. "National Institute of Biotechnology and Department of Microbiology and Immunology, Faculty of Health Sciences, Ben-Gurion University of the Negev, Beersheba, Israel. ${ }^{5}$ School of Chemistry, Faculty of Exact Sciences, Tel Aviv University, Tel Aviv, Israel.

\begin{abstract}
Axonal degeneration is an important determinant of progressive neurological disability in multiple sclerosis (MS). Thus, therapeutic approaches promoting neuroprotection could aid the treatment of progressive MS. Here, we used what we believe is a novel water-soluble fullerene derivative (ABS-75) attached to an NMDA receptor antagonist, which combines antioxidant and anti-excitotoxic properties, to block axonal damage and reduce disease progression in a chronic progressive EAE model. Fullerene ABS-75 treatment initiated after disease onset reduced the clinical progression of chronic EAE in NOD mice immunized with myelin-oligodendrocyte glycoprotein (MOG). Reduced disease progression in ABS-75-treated mice was associated with reduced axonal loss and demyelination in the spinal cord. Fullerene ABS-75 halted oxidative injury, CD11 $\mathrm{b}^{+}$infiltration, and CCL2 expression in the spinal cord of mice without interfering with antigen-specific $T$ cell responses. In vitro, fullerene ABS-75 protected neurons from oxidative and glutamate-induced injury and restored glutamine synthetase and glutamate transporter expression in astrocytes under inflammatory insult. Glutamine synthetase expression was also increased in the white matter of fullerene ABS-75-treated animals. Our data demonstrate the neuroprotective effect of treatment with a fullerene compound combined with a NMDA receptor antagonist, which may be useful in the treatment of progressive MS and other neurodegenerative diseases.
\end{abstract}

\section{Introduction}

Epidemiologic studies show that approximately $85 \%$ of patients with MS initially develop a relapsing-remitting form of the disease $(1,2)$. However, within 10 years, approximately half of those with relapsing-remitting MS (RR-MS) develop secondary progressive MS (SP-MS) (3). In this stage there are few, if any, attacks and the patients progress and accumulate neurological disability. An understanding of the factors associated with disease progression and the development of treatments to control them are crucial goals in MS research. The reversible nature of inflammation and demyelination, which are the hallmarks of RR-MS, does not fully explain why there is a transition to SP-MS. Moreover, treatments which halt the inflammatory response do not always stop disease progression and cumulative neurological disability.

Recent evidence supports the view that axonal degeneration may be a major determinant of progressive neurological disability in patients with MS (4-6). Therefore, the development of new therapeutic approaches designed for neuroprotection, and ultimately at preventing or delaying neurological disability, would be of great benefit for MS patients. Because increased oxidative stress and imbalanced glutamate metabolism can lead to axonal degeneration (7-12) and are associated with relapses and disease progression in MS $(13,14)$, it has been suggested that antioxidants and glutamate receptor (NMDA and AMPA/kainate) antagonists are

Nonstandard abbreviations used: GS, glutamine synthetase; LDH, lactate dehydrogenase; MOG, myelin-oligodendrocyte glycoprotein; RR-MS, relapsing-remitting MS; SP-MS, secondary progressive MS.

Conflict of interest: The authors have declared that no conflict of interest exists. Citation for this article: J. Clin. Invest. 118:1532-1543 (2008). doi:10.1172/JCI33464. agents worthy of investigation for the neurodegenerative component of a disease such as MS (15-20).

In this study we employed a model in which myelin-oligodendrocyte glycoprotein-immunized (MOG-immunized) NOD mice develop chronic progressive EAE to test a $\mathrm{C}_{60}$-fullerene derivative as what we believe to be a novel therapeutic approach to confer neuroprotection and reduce disease progression.

Fullerenes are an allotropic form of carbon which were observed for the first time in 1985 and isolated in 1990 (21). They consist of a molecule composed of 60 carbon atoms that form a hollow sphere 1 nanometer in diameter $(21,22)$. Water-soluble carboxyfullerenes have been described to possess robust neuroprotective effects against excitotoxic, apoptotic, and metabolic insults in cortical cell cultures and, in some in vivo models, have been shown to protect against stroke (23-29). The neuroprotective effect of fullerenes has been attributed to their redox properties and high affinity toward free radicals, as $\mathrm{C}_{60}$ is capable of being reversibly reduced by up to 6 electrons. Moreover, the addition of as many as 34 methyl radicals to a $\mathrm{C}_{60}$ sphere has been reported, leading $\mathrm{C}_{60}$ to be characterized as a "radical sponge" (30). These unique properties of the fullerenes provide an excellent platform for development of novel neuroprotecting agents. Here we used a fullerene derivative termed "ABS-75" (31).

Our strategy was to develop receptor-specific antioxidant therapy. Fullerene ABS-75 is the first example of a water-soluble adamantyl-oligoethyleneglycol-fullerene hybrid, in which NMDA receptor-targeting (antagonist) adamantyl groups are connected to an antioxidant carboxyfullerene moiety via oligoethyleneglycol bridges (see Figure 1A). Adamantane family members are well known NMDA antagonists and have been shown to block NMDA 
A
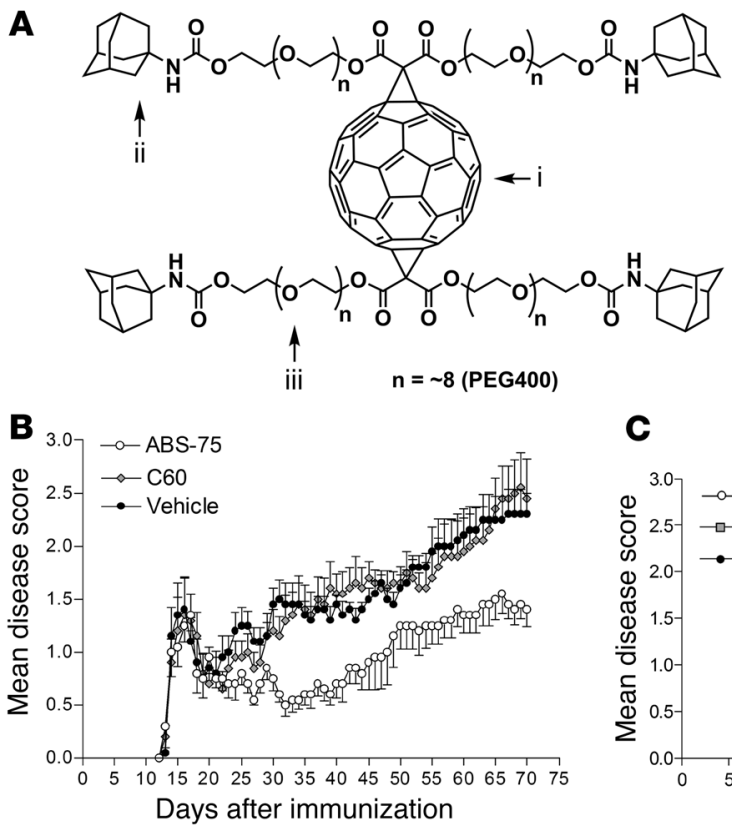

D

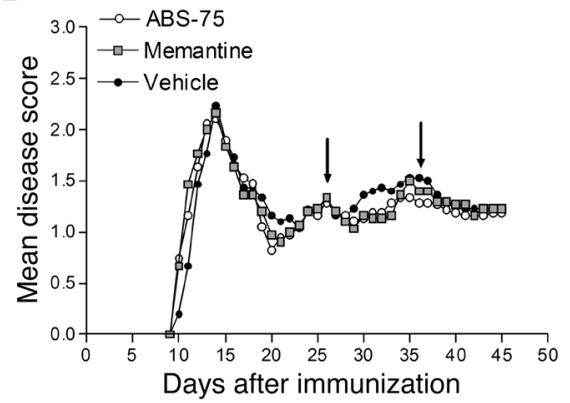

C

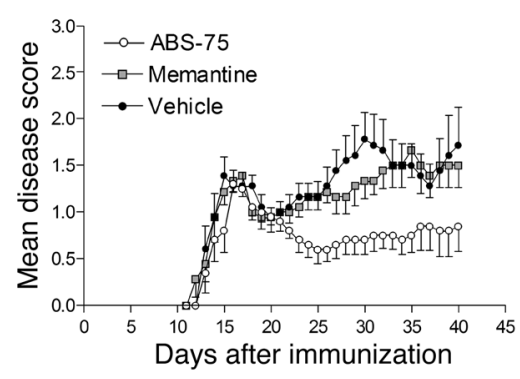

C57BL/6J

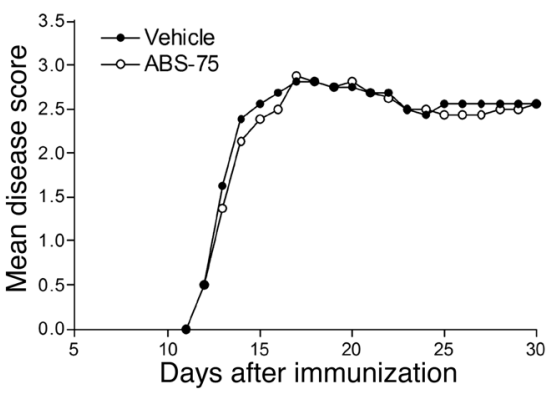

\section{Figure 1}

Fullerene ABS-75 treatment reduces disease progression in secondary progressive EAE. (A) Fullerene ABS-75 consists of the $C_{60}$ fullerene core (i) attached to 4 adamantyl groups (ii) by oligoethyleneglycol bridges (iii). (B) Chronic progressive EAE was induced in 10-week-old NOD mice by subcutaneous immunization with $150 \mu \mathrm{g}$ of $\mathrm{MOG}_{35-55}$ peptide in $4 \mathrm{mg} / \mathrm{ml}$ CFA. Pertussis toxin was given i.v. (150 ng per mouse) at the time of immunization and $48 \mathrm{~h}$ later. Fullerene ABS-75 (30 $\mu \mathrm{g} / \mathrm{kg}$, i.p.) was given daily beginning on day 20. $n=10$ animals per group. Vehicle consisted of $2 \%$ DMSO. (C) Fullerene ABS-75 (30 $\mu \mathrm{g} / \mathrm{kg}$, i.p.) and memantine $(1.5 \mathrm{mg} / \mathrm{kg}$ ) were given daily beginning on day $19 . n=9-10$ animals per group. Vehicle consisted of $2 \%$ DMSO. (D) Left: EAE was induced in 8-week-old SJL mice by s.c. immunization with $50 \mu \mathrm{g}$ of $\mathrm{PLP}_{131-151}$ peptide in $4 \mathrm{mg} / \mathrm{ml} \mathrm{CFA}$. Pertussis toxin was given i.v. (150 $\mathrm{ng}$ per mouse) at the time of immunization and $48 \mathrm{~h}$ later. Fullerene ABS75 (30 $\mu \mathrm{g} / \mathrm{kg}$, i.p.) and memantine $(1.5 \mathrm{mg} / \mathrm{kg})$ were given daily beginning on day 17. $n=15$ animals per group. Arrows indicate relapses following the first attack. Right: Fullerene ABS-75 treatment (30 $\mu \mathrm{g} / \mathrm{kg}$, i.p.) begun 1 day before MOG immunization and done on a daily basis until the end of disease course was not able to ameliorate acute EAE in C57BL/6J mice. $n=8$ animals per group. Disease score measured as follows: 0 , no signs of disease; 1 , loss of tone in the tail; 2 , hindlimb paresis; 3 , hindlimb paralysis; 4 , tetraplegia; 5 , moribund. receptors containing either NR2A, NR2B or NR2D subunits (32). In addition to water solubility, incorporation of biocompatible and flexible oligoethyleneglycol bridges between the 2 functional moieties resulted in improved NMDA receptor affinity, since receptor-binding moieties are not sterically hindered by the fullerene fragment.

ABS-75 is a derivative of carboxyl-functionalized fullerenes, and it has been shown that carboxyl-functionalized fullerenes efficiently cross the blood-brain barrier (33). Furthermore, ABS-75 is considerably more hydrophobic than most carboxyl-functionalized fullerenes, and in contrast to negatively charged carboxyl-functionalized fullerenes, ABS-75 is a neutral molecule. We hypothesized that these features would further enhance the ability of ABS-75 to cross the blood-brain barrier. In addition, a leaky blood-brain barrier is a feature of EAE, which would further facilitate ABS-75 penetration into the brain.

Prior studies of antioxidant targeting focused on delivery of antioxidants to mitochondria $(34,35)$. To our knowledge, although many compounds function as NMDA receptor antagonists (36), targeting an antioxidant functional moiety to the NMDA receptor has not been reported. Here we show that combining the antioxidant properties of fullerene with the anti-excitotoxic properties of an NMDA receptor antagonist in a single molecule was effective in blocking axonal damage and reducing disease progression in a chronic progressive EAE model.

\section{Results}

Fullerene ABS-75 treatment initiated after disease onset reduces EAE progression in the chronic progressive NOD EAE model. In order to investigate the neuroprotective effects of fullerene ABS-75 (Figure 1A), we induced chronic progressive EAE by immunizing NOD mice with $\mathrm{MOG}_{35-55}$. When 10-week-old NOD mice were immunized with $\mathrm{MOG}_{35-55}$ in CFA, the first signs of disease appeared at days 11-12 (peaking at days 16-18), and after partial recovery from this initial acute attack, there was a progressively worsening relapse without full remission. This was then followed by a secondary progressive course characterized by chronic clinical impairment (Figure 1B). This model was chosen because it appeared to be an excellent model for acute MS followed by a progressive chronic phase associated with neurological disability.

Fullerene ABS-75 (30 $\mu \mathrm{g} / \mathrm{kg}$, i.p.) was given daily from day 20 (after the animals recovered from the first peak of disease) to the end of the experiment at day 70. We found that ABS-75 treatment initiated after disease onset reduced EAE progression. Fullerene ABS-75-treated mice had a lower mean clinical score in the mid phase (days 31-34) of the disease course and also by the end of the disease course as compared with vehicle-treated animals (Figure $1 \mathrm{~B}$ and Table 1). Two-way ANOVA also revealed that there was a strong effect of the treatment on disease course. Thus, disease progression was significantly reduced in the fullerene-treated group as compared with vehicle-treated group $(\mathrm{F}=271.68, P<0.0001)$. 


\section{Table 1}

Fullerene ABS-75 treatment reduces disease progression in secondary progressive EAE

$\begin{array}{lcc}\text { Groups } & \text { Disease score above 1.5 } & \begin{array}{c}\text { Mid-phase } \\ \text { Mean disease score } \\ \text { Day } \mathbf{3 1}\end{array} \\ \text { ABS-75 } & 4 / 10 & 0.60^{\mathrm{A}} \\ \mathrm{C}_{60} & 8 / 10 & 1.15 \\ \text { Control } & 8 / 10 & 1.50\end{array}$

\begin{tabular}{|c|c|c|}
\hline \multicolumn{2}{|c|}{$\begin{array}{l}\text { Secondary progressive } \\
\text { Mean disease score }\end{array}$} & Cumulative score \\
\hline Day 38 & Day 70 & Days 21-70 \\
\hline $0.65^{A}$ & $1.40^{\mathrm{A}}$ & $49.8^{A}$ \\
\hline 1.45 & 2.45 & 81.0 \\
\hline 1.40 & 2.30 & 81.6 \\
\hline
\end{tabular}

${ }^{A} P<0.01$ compared with control.

There was interaction between fullerene treatment and disease duration (time), which means that the effect of the treatment was not the same over time. The fullerene treatment seemed to be more effective during the progressive phase of the disease. In addition, cumulative disease score was lower in the fullerene-treated group as compared with the vehicle-treated group (Table 1).

Disease progression in animals treated with the unsubstituted fullerene (fullerene core without the attached NMDA receptor antagonist) was identical to progression of vehicle-treated mice (Figure 1B), demonstrating that the addition of the NMDA receptor antagonist to the fullerene was required for the effect of ABS-75. In addition, treatment with memantine given daily $(1.5 \mathrm{mg} / \mathrm{kg}$ i.p.) beginning at day 20 until the end of disease course did not have an effect on EAE progression (Figure 1C). Taken together, these results demonstrate that combining antioxidant and anti-excitotoxic properties in the same molecule was critical for the effect of ABS-75 in reducing disease progression.

Because NOD mice develop diabetes, we measured blood glucose levels at the end of the disease course and found no evidence of diabetes, which might have confounded our results. Specifically, the blood glucose levels for ABS-75 were $113.17 \pm 16.53 \mathrm{mg} / \mathrm{dl}$, and for control were $115.5 \pm 35.46 \mathrm{mg} / \mathrm{dl}$. These results are consistent with the well-recognized observation that NOD mice immunized with CFA do not develop diabetes $(37,38)$.

We then tested fullerene ABS-75 in 2 traditional relapsing EAE models, the relapsing-remitting SJL model induced with PLP $131-151$ and the monophasic relapsing C57BL/6J model induced with MOG. For the SJL relapsing-remitting model, we initiated treatment with ABS-75 at the same time as in our NOD model (days 17-20) and as a control, we also treated these animals with memantine. As shown in Figure 1D, in SJL mice immunized with PLP $_{131-151}$ the first sign of disease began at days 9-10, and the peak of the first attack occurred at day 14 . The disease score for the first attack was $2-2.5$, which is more severe than what was observed in the NOD MOG model. After remission from the first attack, the animals had 2 subsequent relapses that were much less severe. The animals did not progress in terms of disability, as we observed in the progressive NOD model. Furthermore, as shown in Figure 1D, we did not observe an effect of ABS-75 (or memantine) as measured by clinical progression. Consistent with the clinical data, we did not observe prominent axonal loss in the SJL mouse model as we did in the NOD model (discussed below).

Finally, we tested whether fullerene ABS-75 treatment initiated before immunization had an effect on monophasic MOGinduced EAE in C57BL/6J mice. As shown in Figure 1D, fullerene ABS-75 treatment ( $30 \mu \mathrm{g} / \mathrm{kg}$ i.p.) begun 1 day before MOG immunization and given on a daily basis until the end of disease course did not affect acute EAE in C57BL/6J mice. Thus, fullerene ABS-75 only has an effect on the progressive clinical component unique to the NOD model.

Reduced EAE progression in fullerene ABS-75-treated animals is accompanied by decreased axonal and myelin loss in the spinal cord. Axonal loss and demyelination were assessed by Bielschowsky's silver staining and Luxol fast blue, respectively, in the spinal cord of vehicle- and fullerene ABS-75-treated animals at the end of the experiment and also in animals just after the first peak (day 23). Spinal cords from NOD mice analyzed after the first peak (day 23) showed only mild demyelination and virtually no axonal loss as determined by Bielschowsky's silver staining, demonstrating that the first attack is not associated with significant axonal injury (data not shown). However, after disease progression, we observed pronounced axonal loss (Figure 2A) and demyelination (Figure 2B) in the spinal cord of vehicle-treated animals. In contrast, axonal loss and demyelination were strikingly reduced in fullerene ABS-75-treated animals, consistent with our clinical observations. Neurofilament $\mathrm{H}$ staining, which identifies smaller nerve fibers, also revealed that fullerene ABS-75 treatment was capable of reducing axonal loss after disease progression. As shown in figure $2 \mathrm{C}$, there was a reduction of neurofilament $\mathrm{H}$ staining (percentage of the area within the ventral column of the white matter stained for neurofilament $\mathrm{H}$ ) in controls $(3.96 \% \pm 1.43 \%)$ compared with ABS-75 $(16.13 \% \pm 5.13 \% ; P<0.01)$. We also examined the brain and found perivascular inflammation, as was observed in relapsing EAE models (see Supplemental Figure 3; supplemental material available online with this article; doi:10.1172/JCI33464DS1), which was less than that seen in the spinal cord. We focused our pathological analysis on the lumbar region of the spinal cord because clinical disease in the NOD model (which is restricted to the tail and hindlimbs) is associated with prominent axonal loss in spinal cord but not in the brain.

We then assessed axonal loss in the traditional SJL relapsingremitting mouse model. Unlike the progressive NOD model, we did not observe significant axonal loss in the spinal cord of SJL animals at the end of the disease course (Figure 2D) and there was no effect of treatment with fullerene ABS-75 (data not shown). These results demonstrate that the progressive NOD model is unique both clinically and pathologically as compared with traditional relapsing-remitting EAE models.

Fullerene ABS-75 treatment blocks oxidative stress and diminishes CD11b infiltration into the white matter of the spinal cord in chronic progressive EAE. It was previously reported that fullerene derivatives were able to inhibit macrophage iNOS activity (39). In addition, studies have shown that fullerene increases SOD activity in LPStreated microglia (40). Since inflammation-induced oxidative stress is thought to be involved in the neurodegenerative compo- 

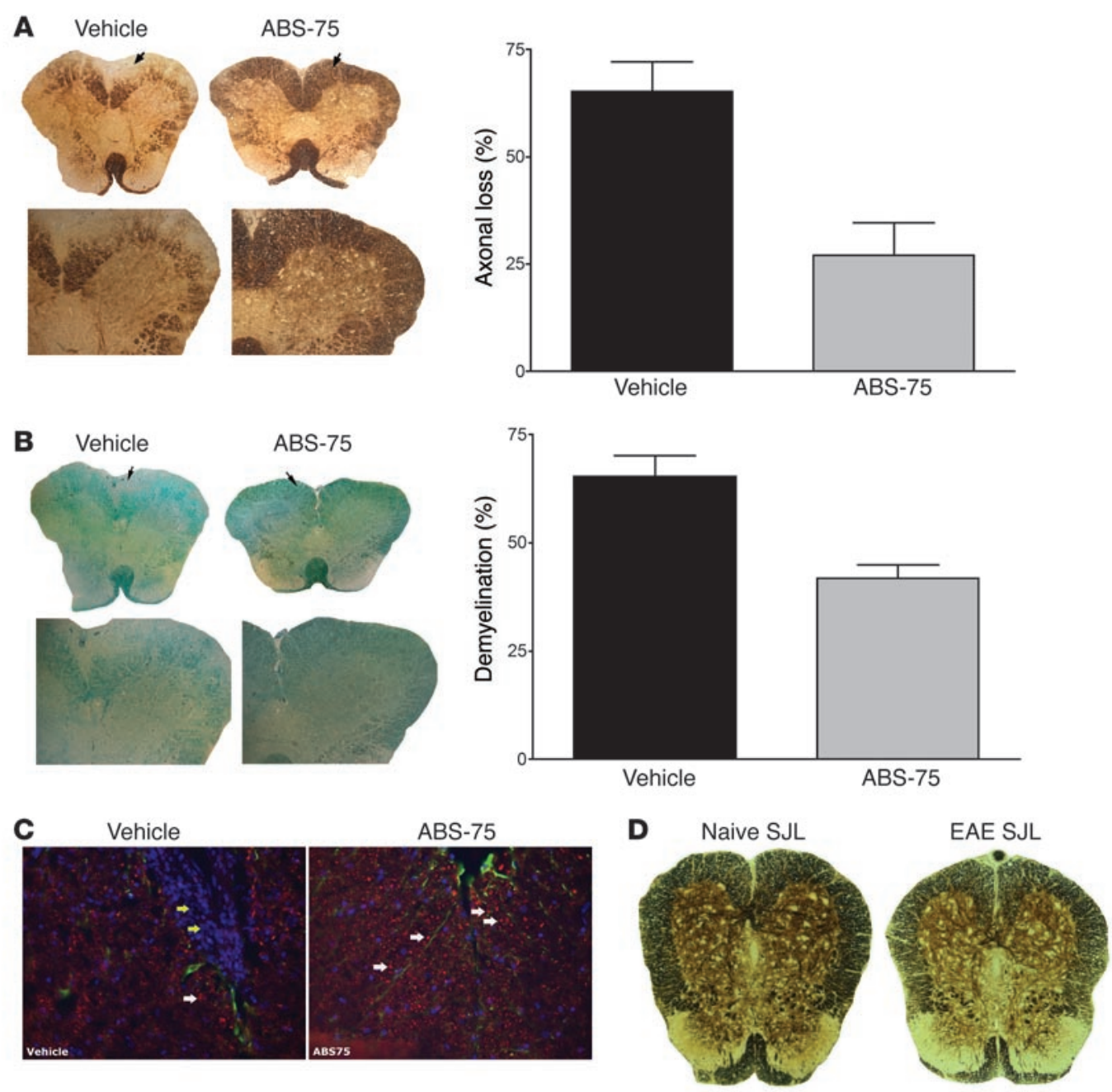

\section{Figure 2}

Fullerene ABS-75 treatment diminishes axonal loss and demyelination in secondary progressive EAE. (A) Bielschowsky's silver impregnation for axons showed a reduction of axonal density in the white matter of the spinal cord (arrows) of a vehicle-treated mouse as compared with ABS-75-treated animal. The axonal pathology analysis was performed after disease progression at the end of the disease course. Original magnification, $\times 50$ (top panels); $\times 100$ (bottom panels). Quantification of stained spinal cord sections by image analysis (ImageJ; NIH) confirmed a reduction in axonal loss when comparing ABS-75- and vehicle-treated animals. $P<0.02$; Student's $t$ test, mean \pm SD. (B) Luxol fast blue staining showed a reduction in the myelin content in the white matter of the spinal cord (arrows) of vehicle-treated mice as compared with ABS-75treated animals. Original magnification, $\times 50$ (top panels); $\times 100$ (bottom panels). Quantification of stained spinal cord sections showed a reduction in demyelination when comparing ABS-75- and vehicle-treated animals. $P<0.01$. Student's $t$ test, mean \pm SD. (C) Neurofilament $H$ staining, which identifies smaller nerve fibers (depicted in red; white arrows) shows reduction in nerve fiber density (percentage of the area within the ventral column of the white matter stained for neurofilament $\mathrm{H}$ ) in controls $(3.96 \% \pm 1.43 \%)$ versus $\mathrm{ABS}-75(16.13 \% \pm 5.13 \%)$. $P<0.01$; Student's $t$ test, mean \pm SD. Nuclei of infiltrating cells stained blue (yellow arrows), and GFAP stained green. Original magnification, $\times 400$. (D) Bielschowsky's silver impregnation for axons showed no significant reduction of axonal density in the white matter of the spinal cord of SJL mice at the end of the disease course as compared with naive animals. Original magnification, $\times 100$.

nent of progressive EAE, we asked whether fullerene ABS-75 would interfere with NO production by activated microglia, which could at least partly explain its ability to reduce disease progression. To address this, we stimulated a murine microglial cell line with peptidoglycan (TLR2 ligand) plus IFN- $\gamma$ in the presence or absence of fullerene ABS-75. As shown in Supplemental Figure 1, in vitro fullerene ABS-75 did not affect TLR2-triggered microglial NO production. However, fullerene ABS-75 treatment initiated after disease onset halted CD11b infiltration into the white matter of the spinal cord in EAE. Hematoxylin and eosin staining performed on spinal cord sections from NOD mice followed for EAE for 70 days revealed an intense inflammatory infiltrate associated with disease progression. To identify the cells comprising the infiltrate, we stained for different markers and found large numbers of CD11 $\mathrm{b}^{+}$cells infiltrating the white matter of the vehicle-treated animals (Figure 3A). Interestingly, only very few $\mathrm{CD}^{+} \mathrm{T}$ cells were observed in the spinal cord of animals with chronic progressive EAE (data not shown). As shown in Figure 3A, numbers of CD11b cells were dramatically diminished in the spinal cord of fullerene ABS-75-treated mice. Because infiltrating CD11b cells may be an 
A
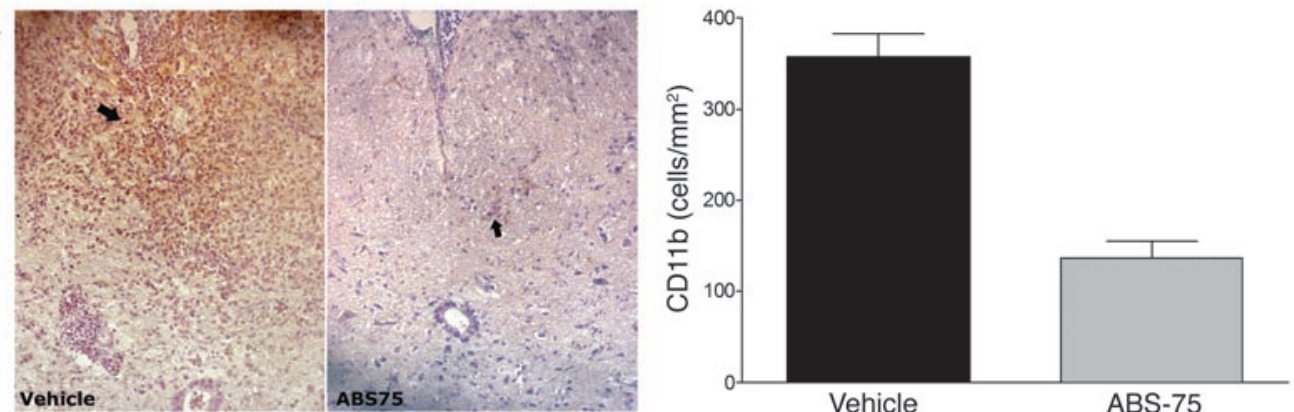

B
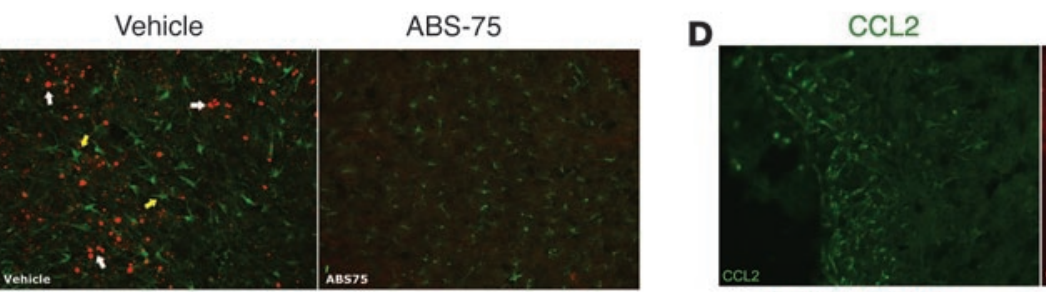

Vehicle

ABS-75

GFAP

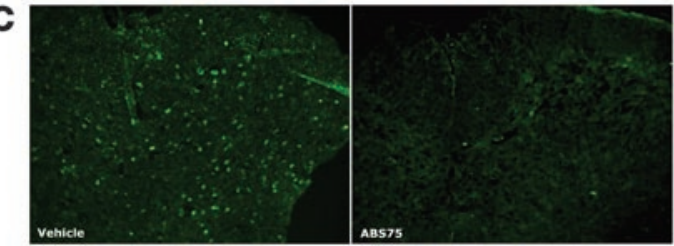

$\mathrm{CCL} 2+\mathrm{GFAP}$

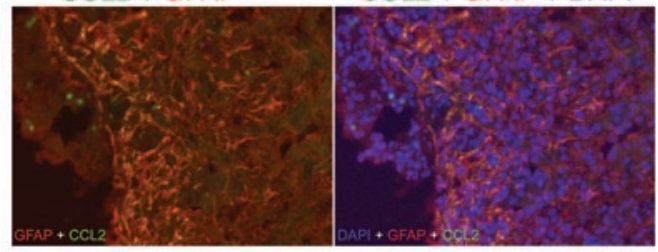

Figure 3

Fullerene ABS-75 treatment halts oxidative injury, blocks CCL2 production by astrocytes, and reduces CD11b infiltration into the spinal cord of secondary progressive EAE mice. (A) CD11b staining revealed large numbers of CD11 b cells infiltrating the ventral column (arrows) of vehicletreated control animals, which was almost absent in mice that received the fullerene ABS-75 treatment. Original magnification, $\times 200$. Quantification confirmed reduced $\mathrm{CD} 11 \mathrm{~b}^{+}$cells infiltrating the spinal cord in ABS-75-treated animals with progressive EAE. $P<0.007$; Student's $t$ test, mean \pm SD. (B) Intense staining for nitrotyrosine (stained red; white arrows) in the spinal cord of vehicle-treated mice after disease progression indicated occurrence of oxidative injury, which was absent in ABS-75-treated animals. The presence of hyperreactive astrocytes (GFAP, stained green, yellow arrows) was associated with oxidative injury in vehicle-treated animals. Original magnification, $\times 200$. (C) CCL2 staining demonstrated a large number of cells expressing CCL2 in the spinal cord of control animals after disease progression, whereas CCL2-positive cells were virtually absent in animals treated with fullerene ABS-75. Original magnification, $\times 100$. (D) Triple staining performed on the spinal cord of control mice revealed high CCL2 expression (green) by astrocytic processes (GFAP, shown in red) in inflamed (blue nuclei of infiltrating cells) regions of the white matter. This demonstrates that in control animals, infiltration of inflammatory cells is associated with astrocytic expression of CCL2. Original magnification, $\times 400$.

important source of molecules causing oxidative damage during progressive EAE, we performed staining for nitrated proteins to investigate whether reduction of CD11b infiltration in fullerenetreated mice was associated with less oxidative injury. Protein tyrosine nitration may result from peroxynitrite action, which is formed by NO reacting with superoxide anion (41, 42). As shown in Figure 3B, while we observed intense staining for nitrotyrosine in vehicle-treated animals, protein tyrosine nitration was virtually absent in the spinal cords from fullerene ABS-75-treated mice. Oxidative injury as measured by nitrotyrosine staining in vehicletreated animals was associated with hyperreactive astrocytes (Figure $3 \mathrm{~B})$. These results demonstrate that fullerene ABS-75 treatment blocked CD11b infiltration and oxidative injury in the spinal cord of EAE NOD mice.

Fullerene ABS-75 treatment is associated with decreased CCL2 expression by astrocytes. Since astrocyte-derived CCL 2 is the main chemokine

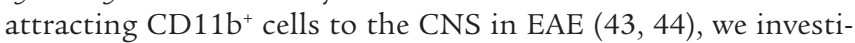
gated the expression of CCL2 following treatment with ABS-75. As shown in figure 3C, we found large numbers of CCL2-expressing cells in vehicle-treated animals after disease progression. In con- trast, CCL2-expressing cells were virtually absent in mice receiving the fullerene ABS-75. Double staining carried out to identify which cells were expressing CCL2 revealed that although there were occasional CD11b cells that were CCL2+ (data not shown), most CCL2expressing cells in the spinal cord of control mice were astrocytes (Figure 3D). The merged image in Figure 3D shows colocalization of CCL2- and GFAP-expressing cells. Association between inflammation and CCL2 production by astrocytes could be observed in the white matter of control mice, where we found a large number of infiltrating cells (blue nuclei) adjacent to proliferating astrocytic processes (GFAP in red) highly expressing CCL2 (Figure 3D). This further suggests that in control animals the CD11b infiltration is associated with astrocyte-derived CCL2.

Next we performed in vitro experiments to test the possibility that fullerene ABS-75 acts directly on astrocytes to block CCL2 expression. Primary cultures of mouse astrocytes were stimulated in vitro with LPS and IFN- $\gamma$ in the presence or absence of fullerene ABS-75 $(1 \mu \mathrm{M})$. The supernatants were collected at $24 \mathrm{~h}$ and measured for CCL2 by ELISA. As shown in Supplemental Figure 2, LPS/IFN- $\gamma$ treatment induced CCL2 production on primary 
A Glutamate $(50 \mu \mathrm{M})$
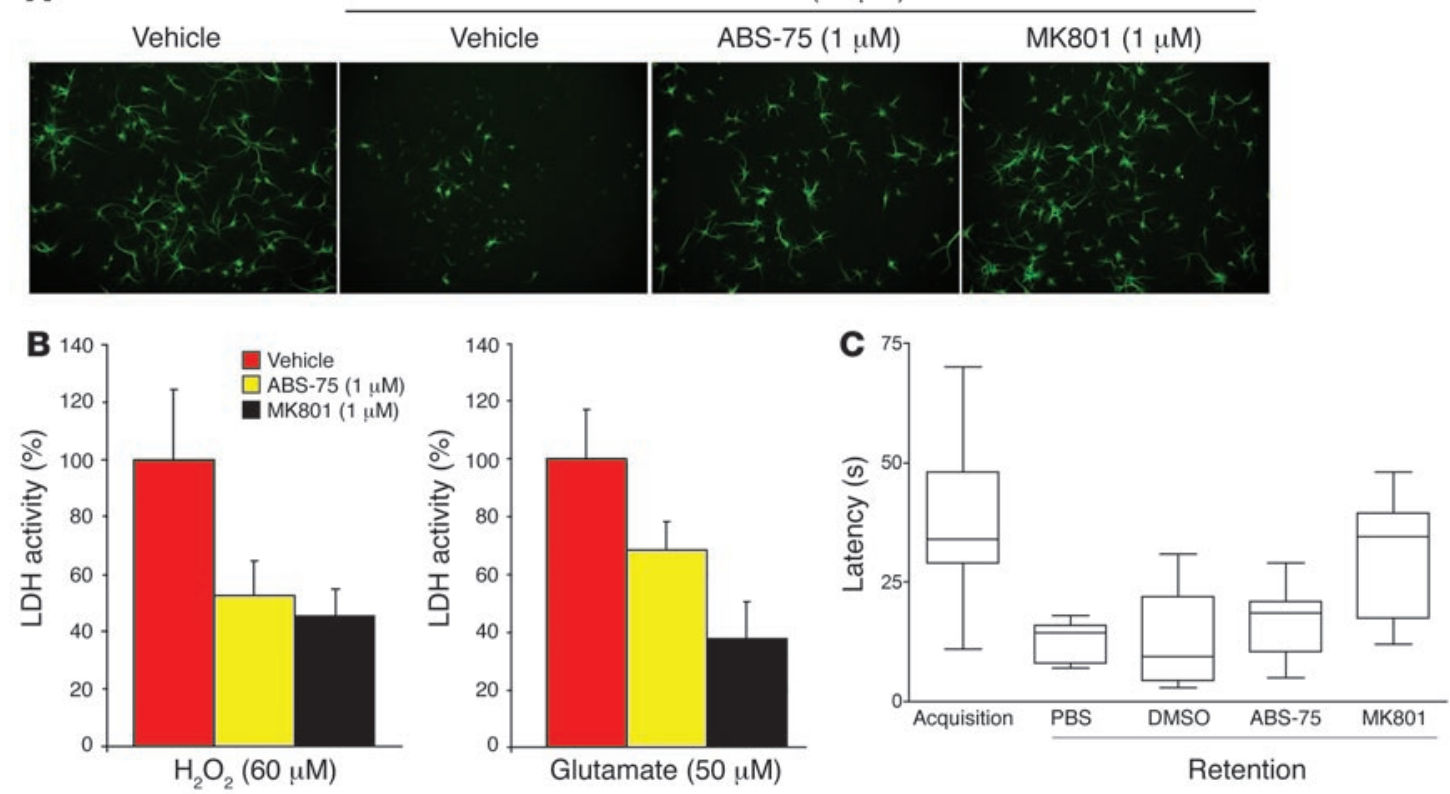

Figure 4

Fullerene ABS-75 protects neurons from oxidative injury and NMDA-dependent glutamate-induced injury but does not impair mouse memory function. Rat cortical neurons (11 DIV) were challenged with either $60 \mu \mathrm{M} \mathrm{H}_{2} \mathrm{O}_{2}$ or $50 \mu \mathrm{M}$ glutamate for $24 \mathrm{~h}$ in the absence or presence of ABS-75 $(1 \mu \mathrm{M})$ or MK801 (1 $\mu \mathrm{M})$. Glutamate- and $\mathrm{H}_{2} \mathrm{O}_{2}$-induced injuries were assessed by (A) MAP-2 staining and (B) LDH activity. The results showed reduced neuronal damage in fullerene ABS-75- and MK801-treated cultures. $P<0.01$, control versus ABS-75 and MK801, and ABS-75 versus MK801. $P<0.01$, control versus ABS-75, and control versus MK801 $\left(\mathrm{H}_{2} \mathrm{O}_{2}\right.$-induced injury). ANOVA followed by post-hoc test, mean \pm SD. (C) Fullerene ABS-75 and MK801 effects on memory function were assessed on a plus maze apparatus. On the first day (acquisition trial), animals were placed at the edge of an open arm and the latency to the first entry in the central area was recorded. On the second day (retention trial), all animals were returned to the arena and the latency to the first transfer onto the central area was recorded. Drugs were administered i.p. $30 \mathrm{~min}$ before the retention trial. All animals moved to the central area in the acquisition trial. In the retention trial, only animals treated with $0.15 \mathrm{mg} / \mathrm{kg}$ MK801 had similar results to those of vehicle mice in the acquisition trial. Animals treated with PBS, $2 \%$ DMSO, or ABS-75 arrived at the central area faster in the retention trial than in the acquisition trial, indicating that, unlike MK801, fullerene ABS-75 does not impair memory function. $P<0.05$, Kruskal-Wallis test followed by Dunn's multiple comparisons test. $n=10$ animals per group. Median (line through each box) \pm SD (error bars) are shown. Original magnification, $\times 130$.

murine astrocytes that was not blocked by ABS-75. These results indicate that although fullerene ABS-75 does not directly act on astrocytes, in vivo treatment downregulates CCL2 expression by astrocytes, which appears to explain the reduction in CD11b infiltration in the spinal cord of fullerene ABS-75-treated animals.

Fullerene ABS-75 protects neurons from oxidative and glutamateinduced injury. Due to imbalanced glutamate metabolism and the consequent increase in its extracellular concentration, glutamateinduced excitotoxicity is thought to be a major cause of axonal damage in MS and EAE. In fact, it has been demonstrated that glutamate receptor antagonists and inhibitors of glutamate transmission were efficient in reducing death of oligodendrocytes and ameliorating clinical EAE (15-18, 45). NMDA-induced neuronal injury has also been shown to induce CCL2 expression by astrocytes in organotypic brain cultures $(46,47)$. Since fullerene ABS-75 bears a moiety known to work as an NMDA receptor antagonist, we asked whether it would be able to protect neurons from glutamate- and $\mathrm{H}_{2} \mathrm{O}_{2}$-induced injury. In order to answer this question, rat cortical neurons were challenged with $60 \mu \mathrm{M} \mathrm{H}_{2} \mathrm{O}_{2}$ or $50 \mu \mathrm{M}$ glutamate for $24 \mathrm{~h}$ in the absence or presence of ABS-75 $(1 \mu \mathrm{M})$ or MK801 $(1 \mu \mathrm{M})$. Glutamate- and $\mathrm{H}_{2} \mathrm{O}_{2}$-induced injury was assessed by MAP-2 staining and lactate dehydrogenase (LDH) activity as an indicator of cell viability. MK801, which is an NMDA receptor antagonist with known neuroprotective properties $(12,48)$, was used as a positive control. As shown in Figure 4, A and B, we found that fullerene ABS-75 possesses neuroprotective properties and inhibits glutamate excitotoxicity. Thus, after glutamate $(50 \mu \mathrm{M})$ and $\mathrm{H}_{2} \mathrm{O}_{2}(60 \mu \mathrm{M})$ challenge, we observed more MAP-2 staining (Figure 4A) and lower LDH activity (Figure 4B) in the neuronal cultures treated with fullerene ABS-75 as compared with control.

Fullerene ABS-75 does not impair memory function. In humans, NMDA receptor antagonists are reported to produce side effects such as drowsiness, hallucinations, psychosis, memory impairment, and vomiting $(49,50)$. In animals, NMDA receptor blockade by MK801 is also accompanied by memory and learning impairment $(51,52)$. The side effects of glutamatergic blockade have led some stroke clinical trials to be discontinued. We did not observe any obvious toxic effects or behavioral changes in our animals, even after treating them for more than 40 days. To directly address memory function, we performed behavioral experiments on ABS-75-treated animals as compared with MK801-treated mice. For these experiments, each mouse was placed at the end of an open arm facing away from the central platform of a plus maze apparatus. The transfer latency, i.e., the time each mouse took to move from the open arm to either enclosed arm was measured on day 1 , and this was the acquisition trial. On day 2, which was the 


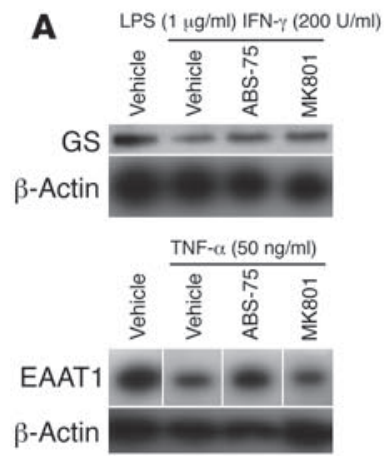

B

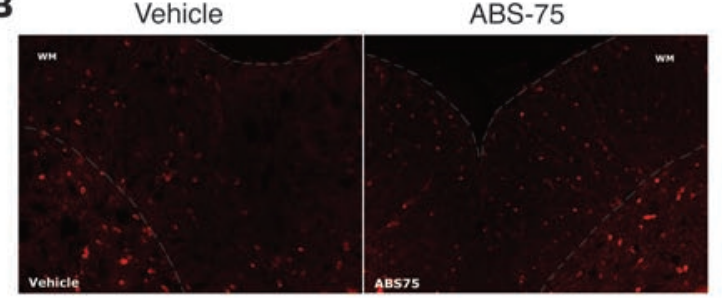

C

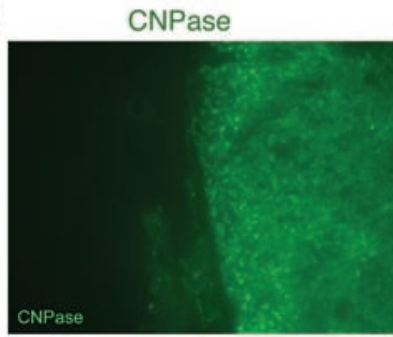

GFAP

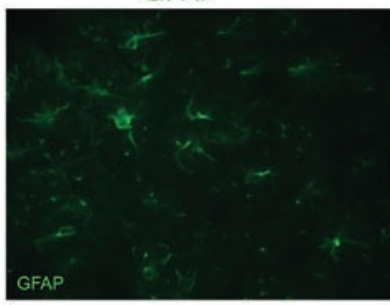

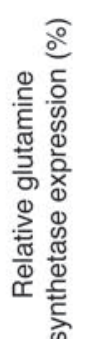

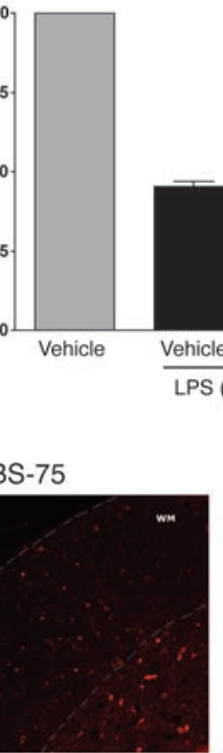

GS

GS

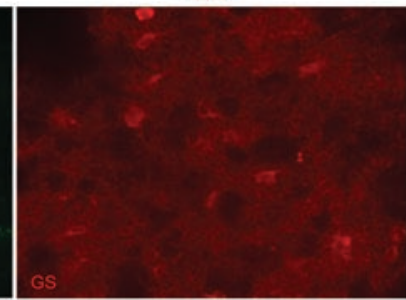

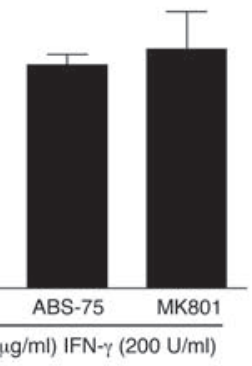
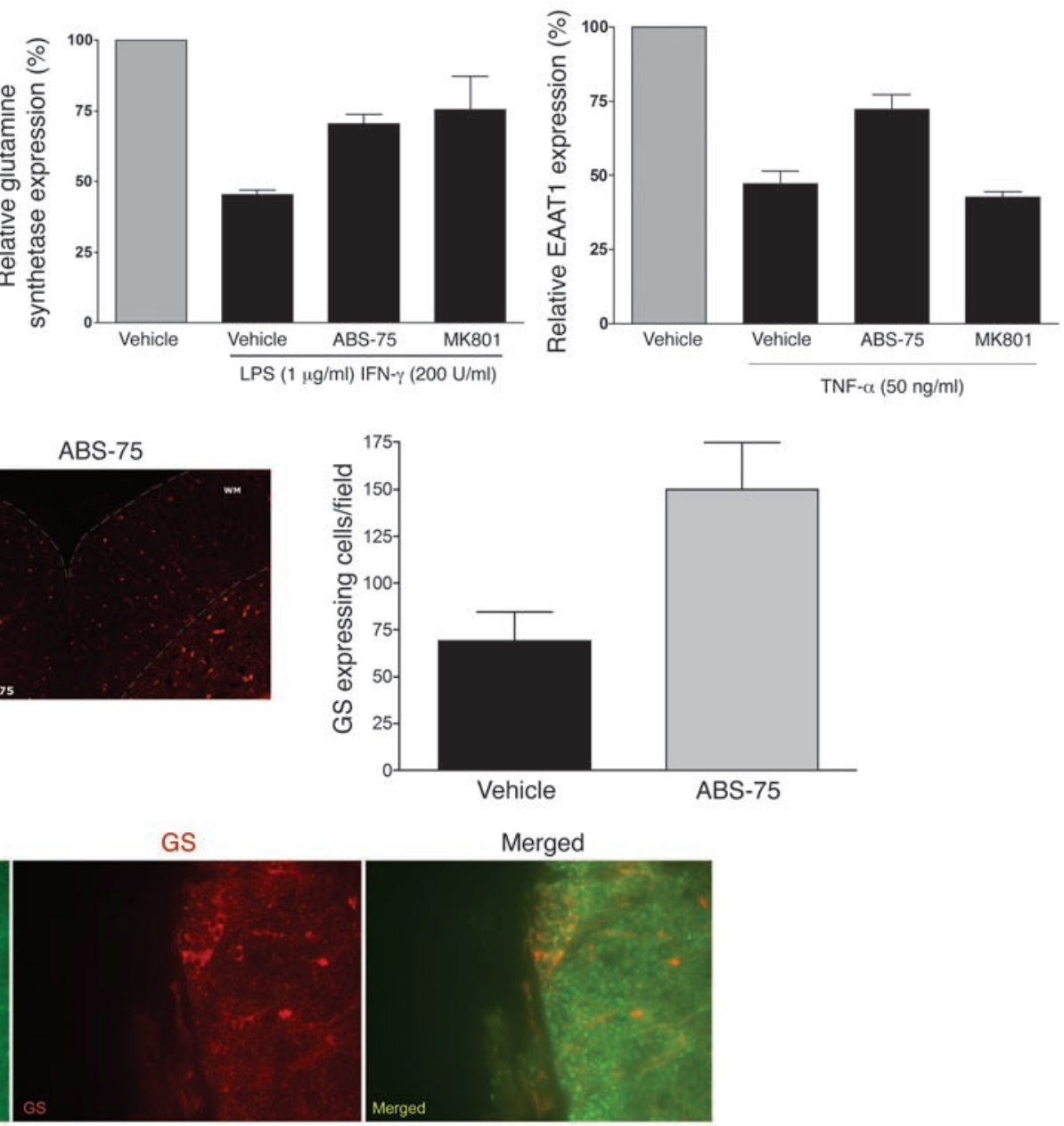

Merged

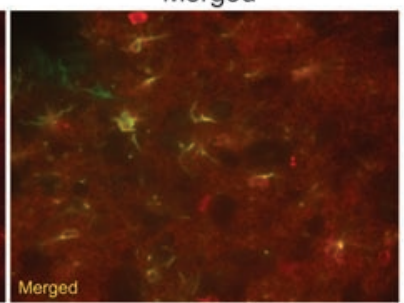

Figure 5

Fullerene ABS-75 rescues the reduced expression of EAAT1 and GS that occurs in astrocytes under inflammatory conditions. (A) Primary mouse astrocytes were stimulated with either TNF- $\alpha(50 \mathrm{ng} / \mathrm{ml})$ or LPS $(1 \mu \mathrm{g} / \mathrm{ml})$ and IFN- $\gamma(200 \mathrm{U} / \mathrm{ml})$ for $24 \mathrm{~h}$ in the absence or presence of ABS-75 $(1 \mu \mathrm{M})$ or MK801 (1 $\mu \mathrm{M})$. GS and EAAT1 expression were assessed by western blot. Quantification was performed using densitometry analysis (ImageJ; NIH) for 2 independent experiments. We found that both ABS-75 and MK801 could reverse the reduced expression of EAAT1 and GS by astrocytes under inflammatory conditions. $P<0.01$, ANOVA followed by post-hoc test, mean \pm SD. (B) Immunohistochemistry performed on spinal cord sections analyzed after disease progression revealed increased GS expression within the white matter of fullerene ABS-75 group as compared with vehicle-treated animals. Quantification by ImageJ confirmed increased numbers of GS-expressing glial cells in the white matter of fullerene ABS-75-treated mice. $P<0.05$, Student's $t$ test, mean \pm SD. (C) Immunohistochemistry performed on spinal cord sections analyzed after disease progression revealed that the cells expressing GS within the white matter were either oligodendrocytes (CNPase positive) or astrocytes (GFAP positive). Original magnification, $\times 200(\mathbf{B}) ; \times 630(\mathbf{C})$.

retention trial, animals were injected either with PBS, $2 \%$ DMSO, MK801 (150 $\mu \mathrm{g} / \mathrm{kg})$, or fullerene ABS-75 (30 $\mu \mathrm{g} / \mathrm{kg})$ and after $30 \mathrm{~min}$ tested for memory function as measured by their transfer latency time. As shown in figure $4 \mathrm{C}$, the transfer latency time observed in PBS- and DMSO-treated mice significantly decreased when comparing acquisition and retention trials, indicating that animals could remember how to escape from the unpleasant situation represented by the open arm. Animals receiving fullerene ABS-75 also presented lower transfer latency time across trials and thus did not differ from PBS- and DMSO-treated mice. 
A
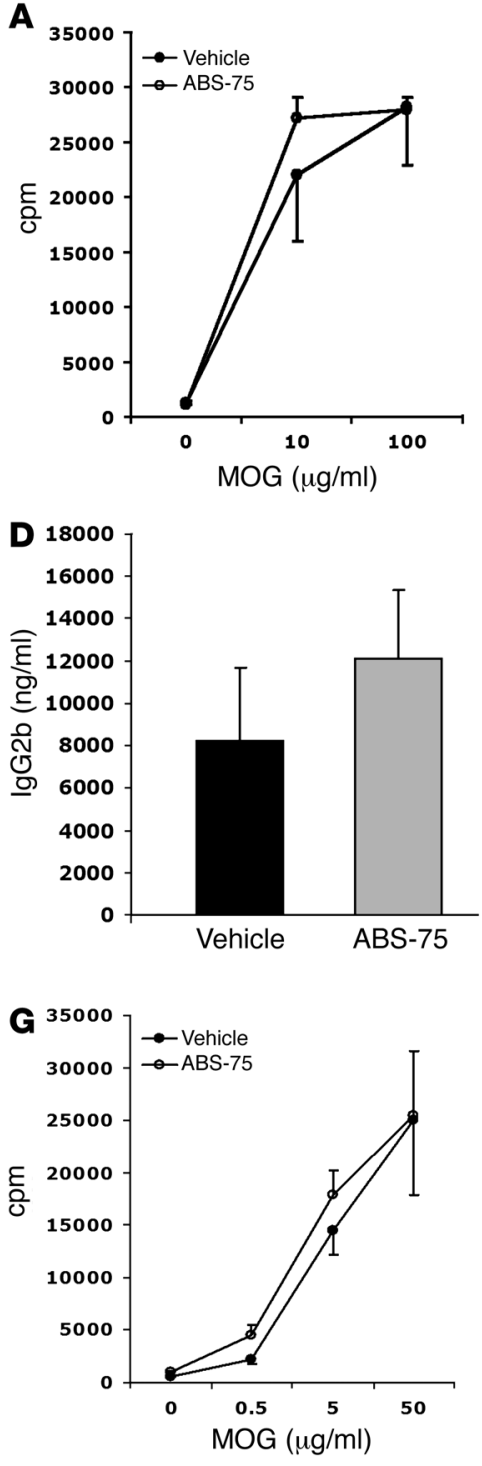

B

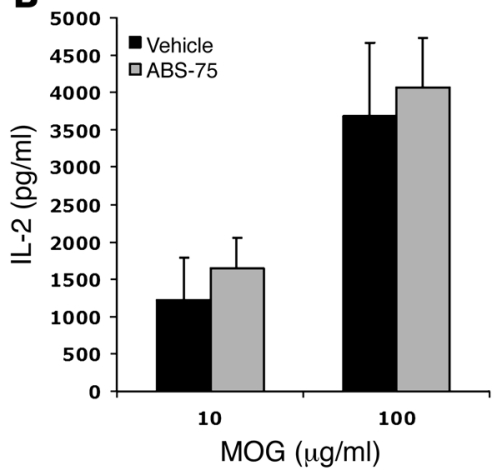

E

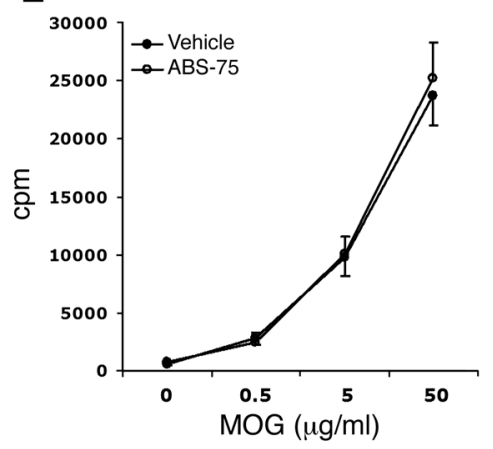

H

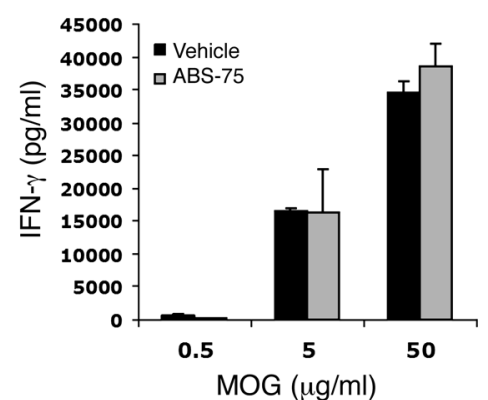

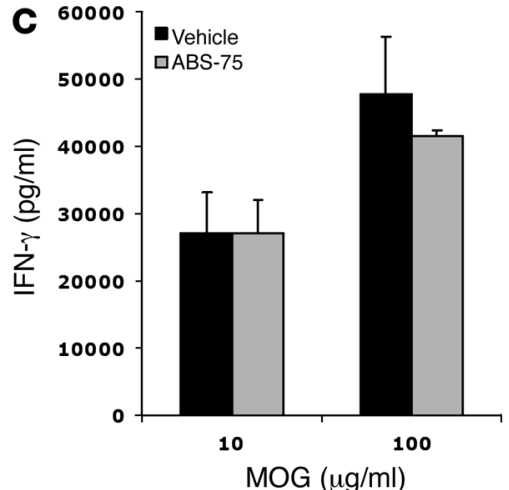

F

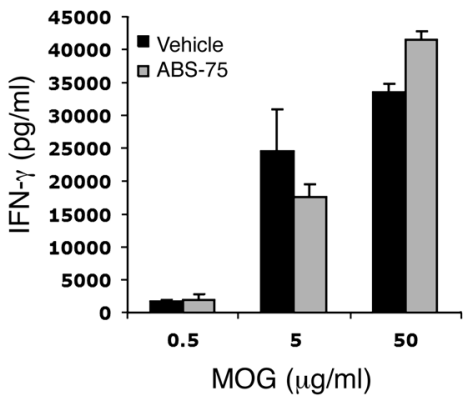

\section{Figure 6}

Fullerene ABS-75 treatment does not interfere with the adaptive compartment of the immune system. (A-C) Splenocytes from vehicle- and ABS-75-treated animals (4 per group) were harvested by the end of disease course and cultivated in the presence of MOG for (A) T cell proliferation, (B) IL-2, and (C) IFN- $\gamma$ production. No differences were observed. (D) Serum collected from vehicle- and ABS-75-treated animals at the end of disease course was tested for MOG-specific lgG2b levels. No differences were observed. (E and F) NOD mice were treated once a day with fullerene ABS-75 (30 $\mu \mathrm{g} / \mathrm{kg}$ i.p.) from day -1 to day 10 after immunization, when draining lymph nodes were harvested to evaluate $T$ cell recall response upon MOG stimulation. No differences were found for $(\mathbf{E}) \mathrm{T}$ cell proliferation and $(\mathbf{F})$ IFN- $\gamma$ production. $(\mathbf{G}$ and $\mathbf{H})$ NOD mice were immunized with MOG, and after 10 days, cells harvested from the draining lymph nodes were stimulated with antigen in the presence or absence of $1 \mu \mathrm{M}$ fullerene ABS-75. In vitro $1 \mu \mathrm{M}$ fullerene ABS-75 did not interfere with $(\mathbf{G}) \mathrm{T}$ cell proliferation and (H) IFN- $\gamma$ production upon antigen-specific stimulation.

In contrast, MK801-treated mice did not improve their performance across acquisition and retention trials and presented increased transfer latency time in the retention trial as compared with PBS- and DMSO-treated animals. These results indicate that, unlike MK801, fullerene ABS-75 does not impair memory function as measured in the animal model.

Fullerene ABS-75 rescues astrocytic glutamate-buffering ability by reversing decreased glutamine synthetase and glutamate transporter expression under inflammatory conditions. Since the glutamate released in synaptic clefts is taken up by astrocytes and then converted into nontoxic glutamine by the glia-specific enzyme glutamine synthetase (GS), astrocytes are considered to be important cells that keep extracellular glutamate concentrations low within the CNS and thereby avoid neurotoxicity $(53,54)$. It has been shown that by enhancing iNOS, inflammatory stimuli lead to a decrease in GS activity (55), which may contribute to imbalanced glutamate metabolism and axonal damage in chronic inflammatory diseases such as MS. Compromised GS activity in astrocytes has been demonstrated in EAE (56). NMDA receptor signaling also has been associated with decreased activity of GS in astrocytes (57). Reduced 
expression of glutamate transporters such as EAAT1 also contributes to the decreased astrocyte glutamate-buffering ability under inflammatory conditions. Indeed, it has been demonstrated that by releasing TNF- $\alpha$, autoreactive T cells reduce EAAT1 expression and glutamate uptake in astrocytes (58). Since fullerene ABS-75 has the potential to quench free radicals such as NO and block NMDA receptors, we investigated whether it can maintain the astrocyte glutamate-buffering ability under inflammatory conditions. Primary murine astrocytes were challenged with either TNF- $\alpha(50 \mathrm{ng} / \mathrm{ml})$ or LPS $(1 \mu \mathrm{g} / \mathrm{ml})$ and IFN- $\gamma(200 \mathrm{U} / \mathrm{ml})$ for $24 \mathrm{~h}$ in the presence or absence of ABS-75 $(1 \mu \mathrm{M})$ or MK801 $(1 \mu \mathrm{M})$. GS and EAAT1 expression were assessed by western blot. As shown in Figure 5A, fullerene ABS-75 reversed the reduced expression of both EAAT1 and GS that occurs under inflammatory conditions and thus maintained astrocyte glutamate-buffering ability.

Since fullerene ABS-75 treatment initiated after disease onset was able to block CD11b infiltration and oxidative injury in the spinal cord of EAE mice, we asked whether the treatment would also prevent downregulation of GS expression by glial cells after disease progression. Immunohistochemistry performed on spinal cord sections analyzed after disease progression revealed reduced GS expression by glial cells within the white matter of control group as compared with fullerene-treated animals (Figure 5B). Double staining demonstrated the expression of GS in both oligodendrocytes (CNPase positive) and astrocytes (GFAP positive) (Figure 5C). The increased frequency of GS-expressing cells was the same for both oligodendrocytes and astrocytes.

Fullerene ABS-75 treatment does not affect adaptive $T$ and $B$ cell responses. In order to determine whether fullerene ABS-75 treatment affected immunologic responses, we first compared antigenspecific $\mathrm{T}$ cell responses of vehicle- and fullerene ABS-75-treated animals at the end of disease course. As shown in Figure 6, A-C, there was no difference in $\mathrm{T}$ cell proliferation or cytokine (IFN- $\gamma$ and IL-2) production upon antigen-specific stimulation between vehicle- and fullerene-treated groups. Also, there was no difference in serum MOG-specific IgG2b levels (Figure 6D). Next, we asked whether fullerene ABS-75 treatment initiated before immunization would interfere with $\mathrm{T}$ cell recall response upon antigenic stimulation. We treated NOD mice once a day with fullerene ABS$75(30 \mu \mathrm{g} / \mathrm{kg}$ i.p.) from day -1 to day 10 after immunization, at which time draining lymph nodes were harvested. As shown in Figure $6, \mathrm{E}$ and $\mathrm{F}, \mathrm{T}$ cell proliferation and IFN- $\gamma$ production upon MOG stimulation were not affected by fullerene ABS-75 treatment initiated before immunization. We also investigated $\mathrm{T}$ cell recall response in the presence of fullerene ABS-75. NOD mice were immunized with MOG, and after 10 days, cells harvested from the draining lymph nodes were stimulated with antigen in the presence or absence of $1 \mu \mathrm{M}$ fullerene ABS-75. Fullerene ABS75 did not interfere with $\mathrm{T}$ cell proliferation and cytokine production upon antigenic-specific stimulation (Figure 6, G and $\mathrm{H}$ ). Altogether, these results demonstrate that fullerene ABS-75 does not affect adaptive $T$ cell autoimmune responses in EAE.

\section{Discussion}

Understanding the mechanisms leading to cumulative neurological disability in patients with MS and further developing effective therapeutic strategies aimed at reducing disease progression is a major goal in MS research. Treatments aiming at only blocking the inflammatory component of the disease have not been successful reducing neurological disability in SP-MS patients $(59,60)$ and in chronic EAE models (61), thus pointing out the necessity for new therapeutic approaches. In the past few years, several studies have contributed to the evolving concept that disease progression in SP-MS is closely related to neurodegeneration and axonal loss (4-6). In this study we used the water-soluble fullerene derivative ABS-75 as a new therapeutic approach aimed at neuroprotection and ultimately at reducing disease progression and neurological disability in a chronic progressive EAE model.

We investigated the effect of ABS-75 in a progressive NOD EAE model induced with MOG peptide. This model is different from the traditional relapsing-remitting SJL and C57BL/6J models, which do not demonstrate the chronic clinical progression or prominent axonal loss in the spinal cord observed in the NOD progressive EAE model. Biozzi mice have been used as a model of chronic EAE, though they do not exhibit clinical progression as in the NOD model (18). The model we have employed is ideally suited to investigate mechanisms and therapies relevant to SP-MS, which involves chronic progressive spinal cord dysfunction. Immune-modulating drugs for relapsing MS that effectively treat traditional relapsing EAE models are not of benefit in the secondary progressive form of MS. No effect of ABS-75 was observed in the SJL and C57BL/6J relapsing models, which do not have a clinical and pathologic progressive component. This observation suggests that different mechanisms are involved in the 2 stages of disease and that therapies with different modes of action are needed to treat SP-MS. Thus, we believe we have not only identified a novel approach for the treatment of SP-MS, but have also characterized a new animal model for the investigation of the secondary progressive form of MS.

Our results demonstrate that combining antioxidant and anti-excitotoxic properties in the same molecule is critical for the effect of ABS-75 in reducing neurodegeneration and disease progression in the NOD EAE model. In fact, treatment with the unsubstituted fullerene (fullerene core without the attached NMDA receptor antagonist) or with memantine alone had no effect on disease progression.

It has been suggested that excitotoxicity due to imbalanced glutamate metabolism and consequent increased extracellular glutamate concentration is one important mechanism by which neurodegeneration occurs in MS (19). Indeed, it has been shown that cerebrospinal fluid glutamate levels are increased during relapses in RR-MS patients and highly increased in SP-MS patients who had an increase of at least 1 point in the EDSS score during the last 6 months as compared with SP-MS patients without any change in the EDSS score $(13,62)$. Accordingly, it has been demonstrated that glutamate receptor antagonists and inhibitors of glutamate transmission were efficient in reducing axonal damage, death of oligodendrocytes, and ameliorating clinical EAE (15-19, 45). Extracellular glutamate levels within the CNS are tightly regulated $(53,54)$, and the increase of these levels is well known to cause neurodegeneration through NMDA receptor signaling $(9,19,63,64)$. Because fullerene ABS-75 bears a moiety that possesses the ability to block NMDA receptors, we tested whether it would directly protect primary neurons in culture from glutamate-induced injury. Our results showed that indeed ABS-75 confers neuroprotection and reduces in vitro excitotoxicity, suggesting this could be one of the mechanisms by which fullerene ABS-75 diminishes axonal loss and reduces disease progression in the NOD EAE model.

Imbalanced glutamate metabolism in MS is likely to occur, at least in part because under inflammatory conditions there is a 
decrease in the expression of glutamate transporters and GS in astrocytes $(55,56,58)$. Glutamate transporters such as EAAT1 are required for glial cells to uptake extracellular glutamate, and GS is a glial-specific enzyme responsible for the conversion of glutamate into nontoxic glutamine. Full expression and activity of EAAT1 and GS are needed for astrocytes to efficiently buffer extracellular glutamate. $(53,54)$. Since increased iNOS activity and glutamate itself have been implicated as being responsible for the downregulation of GS expression by astrocytes under inflammatory insult $(55,57)$, we tested whether fullerene ABS- 75 would rescue the glutamate-buffering ability of primary murine astrocytes in culture. As already mentioned, ABS-75 has a great potential to quench free radicals and block NMDA receptors. Our results show that in vitro fullerene ABS-75 reversed the reduced GS and EAAT1 expression by astrocytes under inflammatory conditions. In addition, immunohistochemistry performed on spinal cord sections analyzed after disease progression revealed that fullerene ABS-75 treatment also rescued GS expression in the white matter of mice with chronic progressive EAE. Altogether, our data suggest that fullerene ABS-75 treatment prevents excitotoxicity directly by blocking NMDA signaling in neurons, and indirectly by correcting imbalanced glutamate metabolism within the CNS, thus avoiding axonal loss and disease progression.

Neuronal injury caused by excitotoxicity has been shown to induce CCL2 production by astrocytes $(46,47)$. Although we did not observe any direct effect of fullerene ABS-75 on LPSinduced CCL2 expression by primary astrocytes in culture, immunohistochemistry demonstrated that fullerene ABS-75 completely blocked CCL2 production in the spinal cord of animals with chronic progressive EAE. We believe it is possible that by blocking excitotoxicity and axonal damage, fullerene ABS-75 treatment was also able to indirectly prevent in vivo CCL2 production by astrocytes. CCL2 has been described as a major chemokine attracting monocytes/macrophages to EAE and MS lesions (43, 44). Thus, it is likely that as a consequence of CCL2 production blockade, fullerene ABS-75 reduced CD11b inflammation in the white matter of chronic progressive EAE mice. Indeed, consistent with the observed reduced CCL 2 expression, much less CD11b ${ }^{+}$ cells were found in the white matter of fullerene ABS-75-treated mice after disease progression.

Monocytes or migrating macrophages comprising the CD $11 \mathrm{~b}^{+}$ population are described as an important source of molecules involved in oxidative injury during EAE and MS $(65,66)$. Because peroxynitrite formed by the reaction between $\mathrm{NO}$ and superoxide anion can lead to protein tyrosine nitration $(41,42)$, we utilized staining for nitrotyrosine as a marker for oxidative injury in chronic progressive EAE. Our results showed that the reduction in the $\mathrm{CD} 11 \mathrm{~b}$ infiltration is accompanied by less oxidative injury in the spinal cord of fullerene ABS-75 treated mice. Thus, besides blocking CD11b infiltration, fullerene ABS-75 treatment also blocks oxidative injury associated with the inflammatory response.

Based on our results, pathogenesis of chronic progressive EAE in the NOD model employed here appears to involve a self-perpetuating cycle in which the initial autoimmune inflammatory response triggers glutamate imbalanced metabolism that causes neuronal injury and axonal loss by excitotoxicity, which in turn induces the production of CCL2 by astrocytes. CCL2 production is responsible for augmenting CD11b inflammation with consequent further increase of oxidative injury, which in turn leads to further imbalance of glutamate metabolism by downregulating
GS and probably glutamate transporters in glial cells. Our results suggest that fullerene ABS-75 is effective at reducing disease progression because it is able to break this self-perpetuating cycle by preventing glutamate-imbalanced metabolism and also by directly blocking NMDA signaling-dependent glutamate-induced injury.

Treatments that block NMDA-dependent glutamatergic transmission can lead to undesirable side effects such as drowsiness, hallucinations, psychosis, memory impairment, and vomiting $(49,50)$. In animals, NMDA receptor blockade by MK801 is also accompanied by memory and learning impairment $(51,52)$. Such side effects have led several selective NMDA antagonists to fail in clinical trials, pointing to the need for agents that are able to modulate glutamatergic transmission but are clinically tolerable. The adamantyl moiety present in fullerene ABS-75 structure is chemically related to memantine, which has been characterized as a therapeutic safe NMDA antagonist due to features such as moderate potency, strong voltage dependence, and fast blocking/unblocking kinetics $(67,68)$. We compared the performance of MK801- and fullerene ABS-75-treated animals on a memory task and observed that, unlike MK801, fullerene ABS-75 does not cause any impairment of memory function. The data suggest that fullerene ABS-75 treatment is not likely to cause side effects due to blockade of physiologically glutamatergic transmission.

We performed a series of experiments to assess possible effects of fullerene ABS-75 on the adaptive compartment of the immune system. We could not detect any in vitro or ex vivo effect of fullerene ABS-75 on T cell function upon antigenic stimulation. Fullerene ABS-75 in vivo treatment begun before immunization also did not interfere with acute EAE in MOG-immunized C57BL/6J mice. Thus, we do not believe fullerene ABS-75 reduces disease progression in chronic progressive EAE by acting on the adaptive compartment of the immune system.

Development of receptor-specific therapy for treatment of CNS disorders is an important therapeutic goal. By targeting an antioxidant molecule to the NMDA receptor, we have developed a new therapeutic strategy for blocking excitotoxicity and oxidative injury in the CNS. This approach prevents axonal loss and reduces neurological disability in a model of SP-MS and is applicable to other neurodegenerative diseases and primary progressive forms of MS as well.

\section{Methods}

Animals and induction of EAE. SJL, NOD, and C57BL/6J mice were purchased from Taconic or The Jackson Laboratory. EAE was induced by subcutaneous immunization of 10-week-old NOD mice or 7-week-old C57BL/6J into the flanks with $200 \mu \mathrm{l}$ of an emulsion containing $150 \mu \mathrm{g}$ of $\mathrm{MOG}_{35-55}$ peptide (MEVGWYRSPFSRVVHLYRNGK; David Teplow, David Geffen School of Medicine, UCLA, Los Angeles, California, USA) and $400 \mu \mathrm{g}$ of Mycobacterium tuberculosis extract H37 Ra (Difco) in incomplete Freund's adjuvant oil. In addition, the animals received $150 \mathrm{ng}$ of pertussis toxin (List Biological Laboratories) i.p. on day 0 and day 2. EAE in SJL mice was induced by immunization with $50 \mu \mathrm{g}$ of $\mathrm{PLP}_{131-151}$ peptide in complete Freund's adjuvant. Clinical signs of EAE were assessed according to the following score: 0 , no signs of disease; 1 , loss of tone in the tail; 2 , hindlimb paresis; 3 , hindlimb paralysis; 4, tetraplegia; 5, moribund. Animals were kept in a conventional pathogenfree facility at the Harvard Institutes of Medicine, and all experiments were carried out in accordance with guidelines prescribed by, and were approved by, the standing committee of animals at Harvard Medical School.

Cytokines and $T$ cell proliferation assays. Splenocytes and lymph node cells were cultured in X-VIVO medium and plated at $5 \times 10^{5}$ cells per well in the 
presence of $\mathrm{MOG}_{35-55}$ for $72 \mathrm{~h}$. For some experiments, lymph node cells were cultured in the presence of $1 \mu \mathrm{M}$ fullerene ABS-75. During the last $16 \mathrm{~h}$, cells were pulsed with $1 \mu \mathrm{Ci}$ of $\left[{ }^{3} \mathrm{H}\right]$-thymidine (PerkinElmer) followed by harvesting on glass fiber filters and analysis of incorporated $\left[{ }^{3} \mathrm{H}\right]$-thymidine in a b-counter (1450 Microbeta, Trilux; PerkinElmer). Supernatants were collect after $40 \mathrm{~h}$ of culture for cytokine measurements by ELISA.

CCL2, EAAT1, and GS expression in primary murine astrocytes. Primary murine astrocytes (>95\% GFAP positive) prepared by standard methodology were stimulated in vitro with TNF- $\alpha(50 \mathrm{ng} / \mathrm{ml})$ or LPS $(1 \mu \mathrm{g} / \mathrm{ml})$ and IFN- $\gamma(100 \mathrm{U} / \mathrm{ml})$ in the presence or absence of $1 \mu \mathrm{M}$ fullerene ABS-75. The supernatants from astrocyte cultures were collected at $24 \mathrm{~h}$ and measured for CCL2 by ELISA. GS and EAAT1 expression was assessed by western blot on astrocyte culture lysates. Protein concentration in each sample was determined using a bicinchoninic acid kit (BCA-1; Sigma-Aldrich), and after dilutions to standardize the samples, the same amount of protein was loaded into a polyacrylamide gel (NuPAGE Novex $12 \%$ Bis-Tris Gel; Invitrogen) for protein separation. The samples were transferred to nitrocellulose membranes (Amersham), and after $1 \mathrm{~h}$ blocking (blocking buffer; Pierce Biotechnology), the membranes were incubated at $4^{\circ} \mathrm{C}$ overnight with the primary antibody (rabbit anti-GS, G2781; Sigma-Aldrich; or rabbit anti-EAAT1, ab416; Abcam). As a secondary antibody, horseradish peroxidase-linked anti-rabbit IgG antibodies (Cell Signaling Technology) were used. Specific signals were visualized using the ECL detection system (Amersham) with Fuji x-ray films (RX NIF). The optical density of the bands was estimated by determining the threshold of visibility using ImageJ software (NIH).

Neuronal injury evaluation. Primary neurons from the rat cortex (N200200; Genlantis) were seeded on 24-well poly-D-lysine/laminin-coated plates at $32 \times 10^{3}$ cells $/ \mathrm{cm}^{2}$ and cultivated in neurobasal/B-27-supplemented medium for 10 days. On day 11 , fully differentiated neurons were challenged either with $50 \mu \mathrm{M}$ glutamate or $60 \mu \mathrm{M} \mathrm{H}_{2} \mathrm{O}_{2}$ for $24 \mathrm{~h}$ either in the presence or absence of the fullerene 1- $\mu \mathrm{M}$ ABS-75. Neuronal damage were assessed by staining for MAP-2 (M4403; Sigma-Aldrich), followed by Alexa Fluor 488-conjugated goat anti-mouse IgG (Invitrogen) and by measuring LDH activity (CytoTox 96 Non-Radioactive Cytotoxicity Assay, G1780; Promega) as an indicator of cell viability.

Microglia cell culture. ATCC EOC 20 murine microglial cell line (CRL2469 ) was cultured in 70\% ATCC DMEM, $10 \%$ fetal bovine serum, and $20 \%$ conditioned medium from LADMAC cell line (CRL-2420) and stimulated with peptidoglycan (TLR2 ligand) plus IFN- $\gamma$ in the presence or absence of different concentrations of fullerene ABS-75. TLR2-triggered microglial $\mathrm{NO}$ production was measured by Griess Reaction utilizing a Total NO/ Nitrite/Nitrate Assay (KGE001; R\&D Systems).

Behavioral experiments. On day 1 (acquisition trial) each mouse was placed at the end of one open arm facing away from the central platform in a plus-maze apparatus. The transfer latency, i.e., the time each mouse took to move from the open arm to either enclosed arm, was measured. Memory retrieval was assessed on the second day (retention trial), when all animals returned to the arena and the transfer latency time was recorded again under the influence of PBS, $2 \%$ DMSO, MK801 $(150 \mu \mathrm{g} / \mathrm{kg})$, or fullerene ABS-75 $(30 \mu \mathrm{g} / \mathrm{kg})$, which were administered i.p. $30 \mathrm{~min}$ before the retention trial.
Immunohistochemistry. Spinal cord tissue samples from MOG-immunized NOD mice at the end of the disease course were collected, fixed in $4 \%$ paraformaldehyde, cryoprotected in $20 \%$ sucrose, and frozen in OCT. Spinal cord frozen sections were cut at $10 \mu \mathrm{m}$ and processed for histological analysis, immunohistochemistry, or immunofluorescence. Axonal loss and demyelination were assessed by Bielschowsky's silver staining and Luxol Fast Blue, respectively. CD11b cells were identified by immunohistochemistry utilizing rat anti-CD11b antibody (MCA711; Serotec), followed by biotinylated goat anti-rat IgG (Jackson ImmunoResearch Laboratories) and standard avidin-biotin immunoperoxidase method (Vector Laboratories). The sections were counterstained with hematoxylin. Immunofluorescence was employed to identify neurofilament $\mathrm{H}-$ positive nerve fibers (rabbit anti-neurofilament 200 antibody, N4142; Sigma-Aldrich), CCL2 expression (goat anti-CCL2 antibody, R\&D systems, AF-479-NA), GS expression (rabbit anti-GS, G2781; Sigma-Aldrich), protein nitration (rabbit anti-nitrotyrosine antibody, N0409; Sigma-Aldrich), oligodendrocytes (chicken anti-CNPase antibody, ab53410; Abcam), and astrocytes (chicken anti-GFAP antibody, PCK-591P100; Covance Research Products Inc.; and rabbit anti-GFAP antibody, G9269; Sigma-Aldrich). Secondary antibodies were purchased from Molecular Probes: Alexa Fluor 594-conjugated goat anti-rabbit IgG (A-11012), Alexa Fluor 488-conjugated goat anti-chicken IgG (A-11039), Alexa Fluor 488-conjugated donkey anti-goat IgG (A-11055), and Alexa Fluor 594-conjugated donkey antirabbit (A-21207). For image analysis, 2 or 3 lumbar spinal cord sections were selected according to regional landmarks from 3 different animals per group. Image analysis was performed using the software ImageJ (NIH). For quantification, level of labeling "threshold" was used as a cut-off to determine positive or negative staining. Data are expressed as mean \pm SD.

Statistics. GraphPad Prism software (GraphPad Software Inc.) was utilized throughout for statistical analysis. Two-way ANOVA analysis and Student's $t$ test were employed to analyze clinical disease score. Quantification for axonal loss, demyelination, CD11b infiltration, neurofilament $\mathrm{H}$, and GS expression were analyzed using the Student's $t$ test. ANOVA was employed to analyze LDH activity. Behavioral experiments were analyzed using Kruskal-Wallis ANOVA test followed by Dunn's Multiple Comparisons test. $P<0.05$ was considered significant.

\section{Acknowledgments}

This work was supported by grants from the National MS Society (to H.L. Weiner) and from Fapesp (grant 04/14128-0; to F.A. CostaPinto) and by the Gruss Lipper Foundation and the Rita Dauber Foundation. We thank Samia Khoury and Yue Wang for the primary astrocyte cultures and Jean Pierre S. Peron for helpful discussions.

Received for publication July 31, 2007, and accepted in revised form January 30, 2008.

Address correspondence to: Howard L. Weiner, Center For Neurologic Diseases, Brigham and Women's Hospital, Harvard Medical School, 77 Avenue Louis Pasteur, HIM 730, Boston, Massachusetts 02115, USA. Phone: (617) 525-5300; Fax: (617) 525-5252; E-mail: hweiner@rics.bwh.harvard.edu.
1. Noseworthy, J.H., Lucchinetti, C., Rodriguez, M., and Weinshenker, B.G. 2000. Multiple sclerosis. N. Engl. J. Med. 343:938-952.

2. Sospedra, M., and Martin, R. 2005. Immunology of multiple sclerosis. Annu. Rev. Immunol. 23:683-747.

3. Weinshenker, B.G., et al. 1989. The natural history of multiple sclerosis: a geographically based study. I. Clinical course and disability. Brain. 112:133-146.

4. Bjartmar, C., Wujek, J.R., and Trapp, B.D. 2003.
Axonal loss in the pathology of MS: consequences for understanding the progressive phase of the disease. J. Neurol. Sci. 206:165-171.

5. Wujek, J.R., et al. 2002. Axon loss in the spinal cord determines permanent neurological disability in an animal model of multiple sclerosis. J. Neuropathol. Exp. Neurol. 61:23-32.

6. van Waesberghe, J.H., et al. 1999. Axonal loss in multiple sclerosis lesions: magnetic resonance imaging insights into substrates of disability. Ann. Neurol. 46:747-754.

7. Brown, G.C., and Bal-Price, A. 2003. Inflammatory neurodegeneration mediated by nitric oxide, glutamate, and mitochondria. Mol. Neurobiol. 27:325-355.

8. Bal-Price, A., and Brown, G.C. 2001. Inflammatory neurodegeneration mediated by nitric oxide from activated glia-inhibiting neuronal respiration, causing glutamate release and excitotoxicity. 
J. Neurosci. 21:6480-6491.

9. Piani, D., Frei, K., Do, K.Q., Cuenod, M., and Fontana, A. 1991. Murine brain macrophages induced NMDA receptor mediated neurotoxicity in vitro by secreting glutamate. Neurosci. Lett. 133:159-162.

10. Werner, P., Pitt, D., and Raine, C.S. 2001. Multiple sclerosis: altered glutamate homeostasis in lesions correlates with oligodendrocyte and axonal damage. Ann. Neurol. 50:169-180.

11. Boje, K.M., and Arora, P.K. 1992. Microglial-produced nitric oxide and reactive nitrogen oxides mediate neuronal cell death. Brain Res. 587:250-256.

12. Chung, R.S., McCormack, G.H., King, A.E., West, A.K., and Vickers, J.C. 2005. Glutamate induces rapid loss of axonal neurofilament proteins from cortical neurons in vitro. Exp. Neurol. 193:481-488.

13. Sarchielli, P., Greco, L., Floridi, A., and Gallai, V. 2003. Excitatory amino acids and multiple sclerosis: evidence from cerebrospinal fluid. Arch. Neurol. 60:1082-1088.

14. Rejdak, K., et al. 2004. CSF nitric oxide metabolites are associated with activity and progression of multiple sclerosis. Neurology. 63:1439-1445.

15. Paul, C., and Bolton, C. 2002. Modulation of bloodbrain barrier dysfunction and neurological deficits during acute experimental allergic encephalomyelitis by the N-methyl-D-aspartate receptor antagonist memantine. J. Pharmacol. Exp. Ther. 302:50-57.

16. Wallstrom, E., et al. 1996. Memantine abrogates neurological deficits, but not CNS inflammation, in Lewis rat experimental autoimmune encephalomyelitis. J. Neurol. Sci. 137:89-96.

17. Pitt, D., Werner, P., and Raine, C.S. 2000. Glutamate excitotoxicity in a model of multiple sclerosis. Nat. Med. 6:67-70.

18. Smith, T., Groom, A., Zhu, B., and Turski, L. 2000. Autoimmune encephalomyelitis ameliorated by AMPA antagonists. Nat. Med. 6:62-66.

19. Groom, A.J., Smith, T., and Turski, L. 2003. Multiple sclerosis and glutamate. Ann. N. Y. Acad. Sci. 993:229-275; discussion 287-288

20. Kanwar, J.R., Kanwar, R.K., and Krissansen, G.W. 2004. Simultaneous neuroprotection and blockade of inflammation reverses autoimmune encephalomyelitis. Brain. 127:1313-1331.

21. Nakamura, E., and Isobe, H. 2003. Functionalized fullerenes in water. The first 10 years of their chemistry, biology, and nanoscience. Acc. Chem. Res. 36:807-815.

22. Bosi, S., Da Ros, T., Spalluto, G., and Prato, M. 2003. Fullerene derivatives: an attractive tool for biological applications. Eur. J. Med. Chem. 38:913-923.

23. Lin, A.M., et al. 2002. Local carboxyfullerene protects cortical infarction in rat brain. Neurosci. Res. 43:317-321.

24. Lin, A.M., et al. 2004. Differential effects of carboxyfullerene on MPP+/MPTP-induced neurotoxicity. Neurochem. Int. 44:99-105.

25. Yang, D.Y., et al. 2001. Systemic administration of a water-soluble hexasulfonated C(60) (FC(4)S) reduces cerebral ischemia-induced infarct volume in gerbils. Neurosci. Lett. 311:121-124.

26. Huang, S.S., et al. 2001. Neuroprotective effect of hexasulfobutylated $\mathrm{C} 60$ on rats subjected to focal cerebral ischemia. Free Radic. Biol. Med. 30:643-649.

27. Dugan, L.L., Gabrielsen, J.K., Yu, S.P., Lin, T.S., and Choi, D.W. 1996. Buckminsterfullerenol free radical scavengers reduce excitotoxic and apoptotic death of cultured cortical neurons. Neurobiol. Dis. 3:129-135

28. Dugan, L.L., et al. 1997. Carboxyfullerenes as neuroprotective agents. Proc. Natl. Acad. Sci. U. S. A. 94:9434-9439.

29. Lin, A.M., et al. 1999. Carboxyfullerene prevents iron-induced oxidative stress in rat brain. J. Neurochem. 72:1634-1640.

30. Krusic, P.J., Wasserman, E., Keizer, P.N., Morton, J.R., and Preston, K.F. 1991. Radical reactions of $\mathrm{C}_{60}$. Science. 254:1183-1185.

31. Bar-Shir, A., Engel, Y., and Gozin, M. 2005. Synthesis and water solubility of adamantyl-OEG-fullerene hybrids. J. Org. Chem. 70:2660-2666.

32. Bresink, I., et al. 1996. Effects of memantine on recombinant rat NMDA receptors expressed in HEK 293 cells. Br. J. Pharmacol. 119:195-204.

33. Yamago, S., et al. 1995. In vivo biological behavior of a water-miscible fullerene: 14C labeling, absorption, distribution, excretion and acute toxicity. Chem. Biol. 2:385-389.

34. Coulter, C.V., Kelso, G.F., Lin, T.K., Smith, R.A., and Murphy, M.P. 2000. Mitochondrially targeted antioxidants and thiol reagents. Free Radic. Biol. Med. 28:1547-1554.

35. Smith, R.A., Porteous, C.M., Coulter, C.V., and Murphy, M.P. 1999. Selective targeting of an antioxidant to mitochondria. Eur. J. Biochem. 263:709-716.

36. Haberny, K.A., et al. 2002. Ontogeny of the Nmethyl-D-aspartate (NMDA) receptor system and susceptibility to neurotoxicity. Toxicol. Sci. 68:9-17.

37. McInerney, M.F., Pek, S.B., and Thomas, D.W. 1991. Prevention of insulitis and diabetes onset by treatment with complete Freund's adjuvant in NOD mice. Diabetes. 40:715-725.

38. Sadelain, M.W., Qin, H.Y., Lauzon, J., and Singh, B. 1990. Prevention of type I diabetes in NOD mice by adjuvant immunotherapy. Diabetes. 39:583-589.

39. Wolff, D.J., et al. 2000. Inhibition of nitric oxide synthase isoforms by tris-malonyl-C(60)-fullerene adducts. Arch. Biochem. Biophys. 378:216-223.

40. Tzeng, S.F., et al. 2002. Effects of malonate C60 derivatives on activated microglia. Brain Res. 940:61-68.

41. Radi, R. 2004. Nitric oxide, oxidants, and protein tyrosine nitration. Proc. Natl. Acad. Sci. U. S. A. 101:4003-4008.

42. Torreilles, F., Salman-Tabcheh, S., Guerin, M., and Torreilles, J. 1999. Neurodegenerative disorders: the role of peroxynitrite. Brain Res. Brain Res. Rev. 30:153-163.

43. Mahad, D.J., and Ransohoff, R.M. 2003. The role of MCP-1 (CCL2) and CCR2 in multiple sclerosis and experimental autoimmune encephalomyelitis (EAE). Semin. Immunol. 15:23-32.

44. Huang, D.R., Wang, J., Kivisakk, P., Rollins, B.J., and Ransohoff, R.M. 2001. Absence of monocyte chemoattractant protein 1 in mice leads to decreased local macrophage recruitment and antigen-specific $\mathrm{T}$ helper cell type 1 immune response in experimental autoimmune encephalomyelitis. J. Exp. Med. 193:713-726.

45. Gilgun-Sherki, Y., Panet, H., Melamed, E., and Offen, D. 2003. Riluzole suppresses experimental autoimmune encephalomyelitis: implications for the treatment of multiple sclerosis. Brain Res. 989:196-204.

46. Katayama, T., et al. 2002. Excitotoxic injury induces production of monocyte chemoattractant protein-1 in rat cortico-striatal slice cultures. Neurosci. Lett. 328:277-280.

47. Szaflarski, J., Ivacko, J., Liu, X.H., Warren, J.S., and Silverstein, F.S. 1998. Excitotoxic injury induces monocyte chemoattractant protein-1 expression in neonatal rat brain. Brain Res. Mol. Brain Res. 55:306-314.

48. Stuiver, B.T., Douma, B.R., Bakker, R., Nyakas, C., and Luiten, P.G. 1996. In vivo protection against NMDA-induced neurodegeneration by MK-801 and nimodipine: combined therapy and temporal course of protection. Neurodegeneration. 5:153-159.
49. Chen, H.S., and Lipton, S.A. 2006. The chemical biology of clinically tolerated NMDA receptor antagonists. J. Neurochem. 97:1611-1626.

50. Gardoni, F., and Di Luca, M. 2006. New targets for pharmacological intervention in the glutamatergic synapse. Eur. J. Pharmacol. 545:2-10.

51. Hlinak, Z., and Krejci, I. 1998. Concurrent administration of subeffective doses of scopolamine and MK-801 produces a short-term amnesia for the elevated plus-maze in mice. Behav. Brain Res. 91:83-89.

52. Hlinak, Z., and Krejci, I. 2002. MK-801 induced amnesia for the elevated plus-maze in mice. Behav. Brain Res. 131:221-225.

53. Hertz, L. 2004. Intercellular metabolic compartmentation in the brain: past, present and future. Neurochem. Int. 45:285-296.

54. Hertz, L., and Zielke, H.R. 2004. Astrocytic control of glutamatergic activity: astrocytes as stars of the show. Trends Neurosci. 27:735-743.

55. Muscoli, C., et al. 2005. The effect of inflammatory stimuli on NMDA-related activation of glutamine synthase in human cultured astroglial cells. Neurosci. Lett. 373:184-188.

56. Hardin-Pouzet, H., et al. 1997. Glutamate metabolism is down-regulated in astrocytes during experimental allergic encephalomyelitis. Glia. 20:79-85.

57. Gorg, B., et al. 2006. Inflammatory cytokines induce protein tyrosine nitration in rat astrocytes. Arch. Biochem. Biophys. 449:104-114.

58. Korn, T., Magnus, T., and Jung, S. 2005. Autoantigen specific $\mathrm{T}$ cells inhibit glutamate uptake in astrocytes by decreasing expression of astrocytic glutamate transporter GLAST: a mechanism mediated by tumor necrosis factor-alpha. FASEB J. 19:1878-1880.

59. Coles, A.J., et al. 1999. Monoclonal antibody treatment exposes three mechanisms underlying the clinical course of multiple sclerosis. Ann. Neurol. 46:296-304.

60. Coles, A.J., et al. 2006. The window of therapeutic opportunity in multiple sclerosis: evidence from monoclonal antibody therapy. J. Neurol. 253:98-108.

61. Pryce, G., et al. 2005. Autoimmune tolerance eliminates relapses but fails to halt progression in a model of multiple sclerosis. J. Neuroimmunol. 165:41-52.

62. Stover, J.F., et al. 1997. Neurotransmitters in cerebrospinal fluid reflect pathological activity. Eur. J. Clin. Invest. 27:1038-1043.

63. Fowler, J.H., McCracken, E., Dewar, D., and McCulloch, J. 2003. Intracerebral injection of AMPA causes axonal damage in vivo. Brain Res. 991:104-112.

64. Rothman, S.M., and Olney, J.W. 1995. Excitotoxicity and the NMDA receptor - still lethal after eight years. Trends Neurosci. 18:57-58.

65. Hill, K.E., Zollinger, L.V., Watt, H.E., Carlson, N.G., and Rose, J.W. 2004. Inducible nitric oxide synthase in chronic active multiple sclerosis plaques: distribution, cellular expression and association with myelin damage. J. Neuroimmunol. 151:171-179.

66. Li, J., Baud, O., Vartanian, T., Volpe, J.J., and Rosenberg, P.A. 2005. Peroxynitrite generated by inducible nitric oxide synthase and NADPH oxidase mediates microglial toxicity to oligodendrocytes. Proc. Natl. Acad. Sci. U. S. A. 102:9936-9941.

67. Parsons, C.G., Danysz, W., and Quack, G. 1999. Memantine is a clinically well tolerated $\mathrm{N}$-methylD-aspartate (NMDA) receptor antagonist--a review of preclinical data. Neuropharmacology. 38:735-767. 68. Parsons, C.G., et al. 1995. Comparison of the poten$c y$, kinetics and voltage-dependency of a series of uncompetitive NMDA receptor antagonists in vitro with anticonvulsive and motor impairment activity in vivo. Neuropharmacology. 34:1239-1258. 